\title{
Substructure of Plasmodesmata
}

\author{
R. L. OVERALL
}

School of Biological Sciences, Macleay Bldg A12, The University of Sydney, New South Wales, 2006, Australia

Fax: +61293514771

Email: roverall@mail.usyd.edu.au

1 Introduction 130

2 Light Microscopy of Plasmodesmata 130

3 Electron Microscopy of Plasmodesmata 131

4 Formation of Primary Plasmodesmata 131

5 Structure of Primary Plasmodesmata 134

5.1 Plasma Membrane 134

5.2 Desmotubule 135

5.2.1 Endoplasmic Reticulum or Proteinaceous Cytoskeleton? 135

5.2.2 Transport through the Desmotubule? 137

5.3 Cytoplasmic Annulus 137

5.3.1 Interpretation of the Mottled Layer:

a Possible Role for Actin 138

5.3.2 Spokes Connect the Mottled layer

with the Plasma Membrane 139

5.3.3 Dimensions and Regulation of the Cytoplasmic Annulus 140

5.4 Specializations of the Wall Sleeve 142

5.4.1 Plasmodesmata are Firmly Anchored to the Surrounding Wall 142

5.4.2 Callose 142

5.4.3 Pectin 143

5.4.4 Putative Extracellular Sphincters 143

6 Concluding Remarks 145

References 145 


\section{Introduction}

Plasmodesmata now appear to be highly dynamic structures which are able to select and transport even large molecules from cell to cell (for review, see Zambryski 1995). It has become crucial to understand the substructural architecture of plasmodesmata and the molecular interactions that control the intercellular passage of molecules and viruses. Our images of plasmodesmal structure have been necessarily static, leading to the generation of similarly static models of their architecture. The present challenge is to incorporate the emerging data on the molecular composition and operation of plasmodesmata into a dynamic model of their substructure.

\section{$2 \quad$ Light Microscopy of Plasmodesmata}

The first observations of plasmodesmata at the level of the light microscope are attributed to Tangl (1879). The ensuing early observations which involved swelling of the wall and, in some cases, staining were reviewed in the first book on plasmodesmata (Robards 1976; Carr 1976). The structures visible in the light microscope were no doubt pit fields or wall modifications associated with plasmodesmata. In recent years, various fluorescent probes have been used to label pit fields and, in combination with confocal laser scanning microscopy, to identify the location of single plasmodesmata. In plasmolyzed onion epidermal cells, staining of the endoplasmic reticulum (ER) with the fluorochrome $\mathrm{DIOC}_{6}$ colocalizes with aniline blue-induced fluorescence of callose in the cell walls, presumably at pit fields (Oparka et al. 1994). Rhodamine phalloidin labelling results in fluorescent spots on the walls, showing actin in pit fields of epidermal peels or individual plasmodesmata in tobacco suspension culture cells (White et al. 1994). Immunofluorescence of callose (Fig. 1), cytoskeletal elements
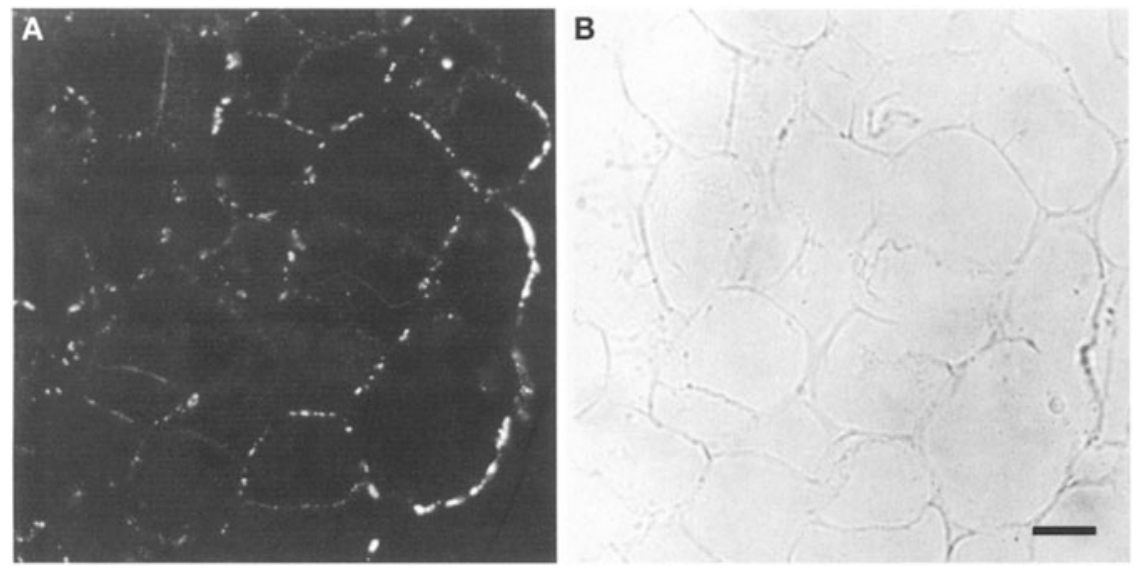

Fig.1A, B. Immunofluorescence localization of callose in mature cells of cauliflower florets. A Epifluorescence image of a section labelled with an antibody to callose showing punctate label of the cell walls consistent with the distribution of plasmodesmata. B Corresponding interference contrast image. Bar $5 \mu \mathrm{m}$. (Unpublished image kindly provided by L. M. Blackman.) 
(Blackman and Overall 1998) and pectin (Casero and Knox 1995) have shown punctate labelling consistent with the distribution of plasmodesmata. Recently, the viral "movement protein" which facilitates the movement of viruses through plasmodesmata has been fused to the green fluorescent protein (GFP) and expressed as an expression vector or from the whole virus (for review, see Oparka et al. 1996). The GFPmovement protein fusion localizes spectacularly to plasmodesmata and has even been seen sandwiched between paired callose platelets at the ends of single plasmodesmata (Oparka et al. 1997). While these various fluorescent tags have been useful in identifying molecular components of plasmodesmata and giving an indication of their distribution at the light microscope level, ultimately it is necessary to use the electron microscope to confirm that the labelled structures are indeed plasmodesmata.

\section{Electron Microscopy of Plasmodesmata}

The small size of plasmodesmata has meant that their substructure has been visible only with the electron microscope. They were first observed in the electron microscope 40 years ago (Buvat 1957), and the main features of images obtained since then have been remarkably consistent. The features visible in Fig $2 \mathrm{~A}-\mathrm{E}$ were clearly present in the images of Robards (1976). Even different tissue preparation techniques e.g. freeze substitution (Fig. 2E; Ding et al. 1992) yielded similar images. In a transverse section in the centre of a plasmodesma, in general, there is a central black dot of $3 \mathrm{~nm}$ in diameter surrounded by an electron-lucent ring $2.2 \mathrm{~nm}$ wide and further electrondense material surrounded by a mottled layer of electron-lucent and electron-dense material. This mottled layer may abut directly onto the alternating outer rings of electron density, electron lucence, and electron density of the plasma membrane (Fig. 2B). Alternatively, there may be an electron-lucent region between the mottled layer and the plasma membrane which is traversed by electron-dense spokes (Fig. 2C). Often, when tannic acid has been added to the fixative, additional electron-dense material may be observed outside the plasma membrane (Fig. 3).

However, surprisingly, the interpretation of the substructure from these images is still under debate. One difficulty is that the width of the plasmodesma channel is comparable to the thickness of a thin section for electron microscopy, so that the components of the structure in longitudinal view are always superimposed within the section. In transverse view, up to one third of the plasmodesma may be superimposed in one section. In addition, our understanding of the substructure of plasmodesmata almost certainly has been stilted by artefacts induced during tissue preparation and difficulties in interpretation of staining patterns. Our interpretation of images from electron microscopy must necessarily be cautious and new technologies to avoid such artefacts must continually be sought.

\section{Formation of Primary Plasmodesmata}

Primary plasmodesmata are formed at cytokinesis as endoplasmic reticulum (ER) is trapped within the fusing Golgi-derived vesicles of cell wall material forming the cell plate (Hepler 1982; Staehelin and Hepler 1996). Nothing is known of how plasmodes- 


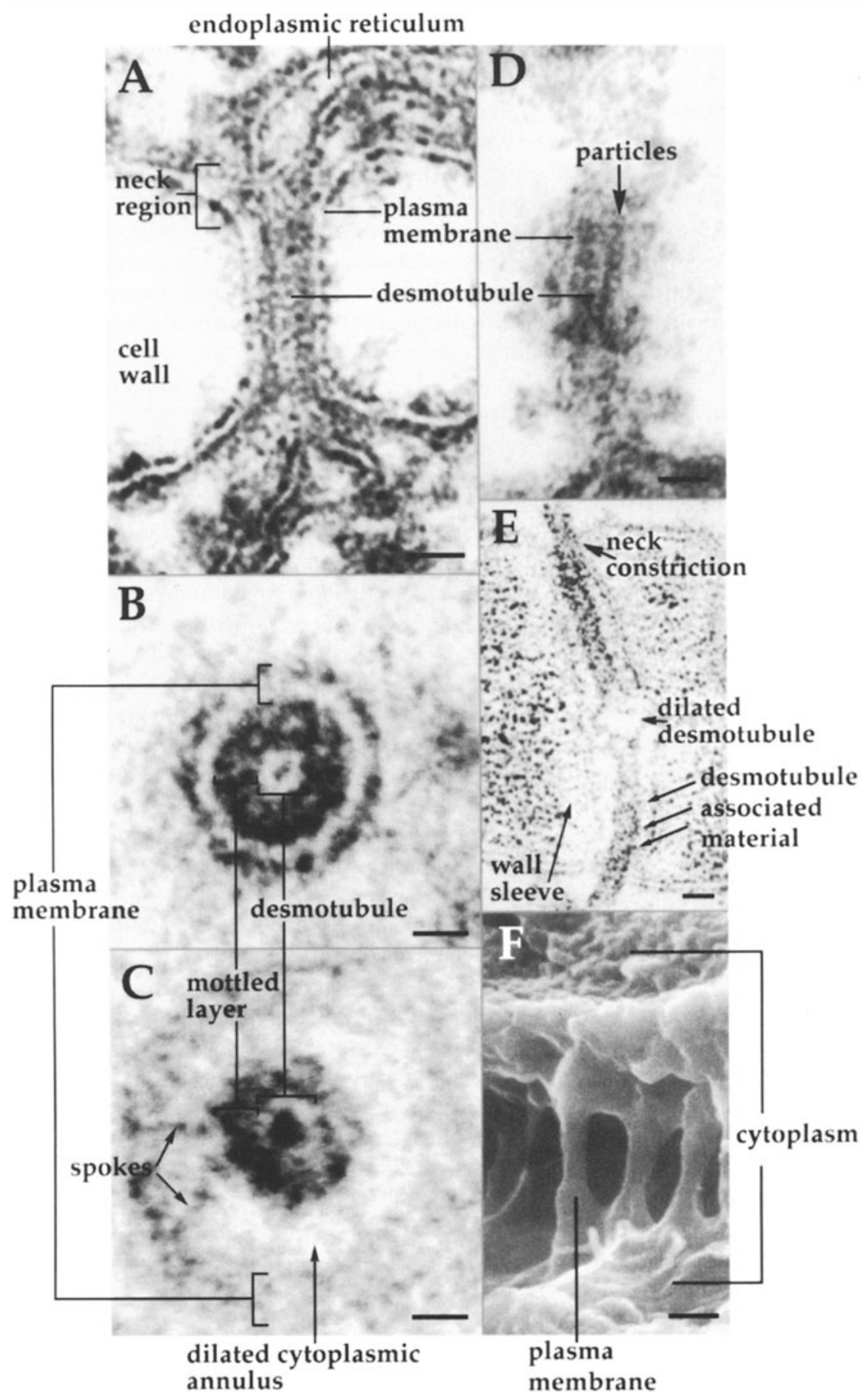


Fig2A-F. Plasmodesmata as seen in the electron microscope. In both longitudinal (A, D-F) and transverse $(B, C)$ images, the plasma membrane delimits the plasmodesma. The central axial structure - the desmotubule (A-E) - is continuous with the endoplasmic reticulum (ER) of neighbouring cells; it consists of tightly furled ER membrane, although occasionally it dilates to form a lumen (E). A mottled layer outside the desmotubule (A-E) may include negatively stained electron-lucent particles (B-D). Sometimes there is a dilated cytoplasmic annulus outside the mottled layer (C, E), but this is often restricted at the neck region as shown in $\mathbf{E}$; spokes traverse the cytoplasmic annulus (C). A-D are sections from chemically fixed material with tannic acid included in the fixative, $\mathbf{E}$ is freeze-substituted material and $\mathbf{F}$ is an image produced by high-resolution scanning electron microscopy. All images are from Azolla roots, except for $\mathrm{C}$ which is from Egeria densa leaves treated with the callose synthesis inhibitor DDG, and E from a Berberis floral nectary. Bars $25 \mathrm{~nm}(\mathrm{~A}, \mathrm{D}, \mathrm{E}), 10 \mathrm{~nm}(\mathbf{B}, \mathbf{C})$, and $75 \mathrm{~nm}(\mathbf{F})$. (A, B, and D Overall et al. 1982; C J. Radford in Overall and Blackman 1996); F unpublished image kindly provided by P. Vesk and M. Vesk
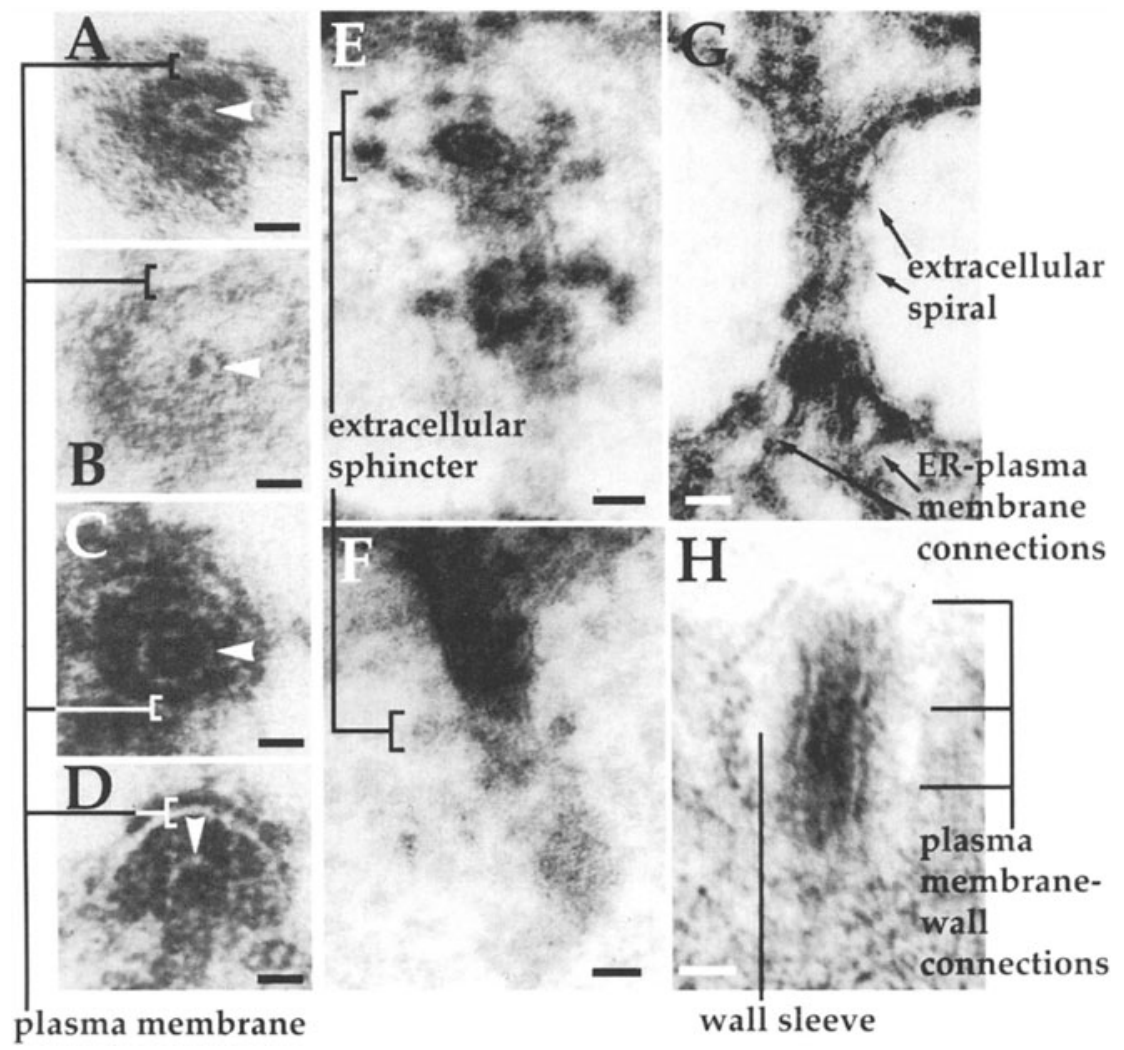

Fig 3A-H. Features of the neck region and wall sleeve of plasmodesmata seen in the electron microscope. At the neck region, the plasma membrane and the tightly furled ER membrane (arrowheads) of the desmotubule funnel out into the cytoplasm and ER lumen, respectively (A-D). Electron-dense particles encircle the necks of some plasmodesmata forming putative extracellular sphincters (E-G), and electron-dense material may also spiral down the length of some plasmodesmata (G). At the neck region, the plasma membrane may be connected to the ER (G) and also to the surrounding cell wall (H) with electron-dense spokes. All images are of chemically fixed material with tannic acid included in the fixative. A-D and $\mathbf{G}$ are from Azolla roots, $\mathrm{H}$ from isolated walls of Azolla roots, $\mathbf{E}$ from Nephrolepsis exaltata rhizomes and $\mathbf{F}$ from Spirodela oligorhiza roots. Bars $10 \mathrm{~nm}(\mathbf{A}-\mathbf{D}), 20 \mathrm{~nm}(\mathbf{E})$ and 25 nm (E-H). (A-D and G, H Overall et al. 1982; E, F Badelt et al. 1994) 
mal components, including specialized surrounding wall structures, are assembled in forming plasmodesmata. Hepler (1982) found that ferric chloride used as a stain for the lumen of the ER was excluded from the ER near the point of cell plate fusion with parent walls, suggesting that the ER forms a tightly furled cylinder as it passes through the plasmodesma. It is not known if the tightly furled cylinder of ER is formed because it becomes squeezed by the fusing vesicles, or if it is formed by a more active process. Proteins of the dynamin family form helical structures around tubular templates and are involved in pinching off membranes ( $\mathrm{Gu}$ and Verma 1996). It may be that the dynamin-like molecule, phragmoplastin, which has been identified in the forming cell plate ( $\mathrm{Gu}$ and Verma 1996) plays a role in the production of this tightly curled ER membrane tube.

In some systems there appears to be precise control over the frequency of plasmodesmata put down during cytokinesis. For example, it has been shown that there is no formation of plasmodesmata in existing walls in Azolla roots, but rather all plasmodesmata are inserted at cytokinesis (Gunning 1978). Remarkably, the number of plasmodesmata put down in a given wall accurately predicts the cell wall expansion that will take place during development for the correct final frequency to be obtained. New cell walls put in during the development exactly mimic the frequency of plasmodesmata in other walls in that region. Nothing is known of how this process is controlled, but recently short-term treatment with the putative cellulose biosynthesis inhibitor dichlobenil (DCB) has led to formation of thickened cell plates with increased callose and increased plasmodesmal density (Vaughn et al. 1996). In contrast, another putative cellulose biosynthesis inhibitor, isoxaben, leads to the formation of thinner plates with less than $20 \%$ of the callose of controls. The ER lines up along the cell plate as in controls, but the plasmodesmata do not form at most of these sites (K. C. Vaughn pers. comm.). In combination, these data point to a role for cell plate callose in the formation of plasmodesmata. Further characterization of the responses of plant cells to these drugs may give us some insight into the processes involved in the deposition of a precise number of plasmodesmata at cytokinesis.

Simple plasmodesmata formed at cytokinesis generally are straight tubes traversing the wall. The adjoining cells must expand in a precisely coordinated fashion in order for the plasmodesmata to remain intact and straight. It is interesting to note that in the walls of BY-2 tobacco suspension culture cells which are habituated to DCB there is little cellulose, but large amounts of pectin, and the plasmodesmata are displaced or oriented more obliquely to the surface of the wall than usual (N. Durso and K. C.Vaughn pers. comm.). It may be that cellulose microfibrils or specific interactions with them are important for the formation and maintenance of straight plasmodesmata or precise coordination of expansion of neighbouring cells.

\section{$5 \quad$ Structure of Primary Plasmodesmata}

\subsection{Plasma Membrane}

The plasma membranes of neighbouring cells, and theoretically the whole plant, are continuous through the plasmodesmata (Fig. 2A, E, F). Its trilaminar structure can be seen clearly around the outside of the plasmodesma cut in transverse section (Fig. 2B). 
At the neck region of the plasmodesma, the plasma membrane funnels out and may appear diffuse in transverse section (Fig. 2C). However, there must be a molecular sieve to prevent diffusion of membrane components between adjacent cells, so that individual cell identity markers can be maintained. Proteins commonly found in the plasma membrane are excluded from the regions around the plasmodesmata (for review, see Robards and Lucas 1990; Fleurat-Lessard et al. 1995). Grabski et al. (1993) demonstrated that plasma membrane lipids are not able to diffuse between neighbouring cells. The membrane-associated particles observed at the necks of plasmodesmata using freeze-fracture electron microscopy (Willison 1976; Thomson and Platt-Aloia 1985) may act as a sieve for molecular diffusion in the plasma membrane through the plasmodesmata. Thomson and Platt-Aloia (1985) have also shown that the external face of the plasma membrane of plasmodesmata in Tamarix salt glands is devoid of intramembranous particles, and they suggest that this half of the membrane might be in a gel, or non-fluid state. In contrast, they have shown that the protoplasmic face of the plasma membrane within the plasmodesma is enriched in particles in agreement with the suggestions of Ding et al. (1992) and Botha et al. (1993) on the basis of computer-enhanced images of plasmodesmata. However, it should be noted that while these workers have identified the inner electron-dense layer of the plasma membrane as being wider than the external electron-dense layer, it has not been noted by numerous other workers and, indeed, Schulz (1995) specifically notes that this is not the case in plasmodesmata of Pisum sativum roots.

\subsection{Desmotubule}

\subsubsection{Endoplasmic Reticulum or Proteinaceous Cytoskeletal Element?}

There is a central axial structure, termed the desmotubule, inside the plasmodesma that is generally believed to be connected to the ER in the adjoining cells. There is continuing controversy over the details of its structure. Most models now interpret the desmotubule as being tightly furled ER, as was first suggested by Lopez-Saez et al. (1966), but others have interpreted the structure as more like a cytoskeletal element.

Initially, it was suggested that the plasmodesmata form as remnants of the spindle fibres, and the similarity of the mottled layer in plasmodesmata to a microtubule led a number of workers in the 1960s to suggest the desmotubule was actually a cytoplasmic microtubule (for review, see Jones 1976). This idea was dismissed rather quickly because plasmodesmata with desmotubules are seen in some algal walls which are formed with the microtubules parallel to the forming cell plate (Marchant 1976) and the desmotubules do not always maintain tubular form (O'Brien and Thimann 1967). It is interesting that tubulin has been found in protein extracts of walls containing plasmodesmata and not in extracts of walls without plasmodesmata, but the tubulin could not be convincingly localized to plasmodesmata using immunogold cytochemistry (Blackman and Overall 1998). Robards (1971) modified the cytoplasmic microtubule idea such that the desmotubule was a hollow tube continuous with the lumen of the ER and bounded by a proteinaceous wall identified as the mottled layer seen in electron micrographs. The inner electron-lucent ring was interpreted as a lumen continuous with the lumen of the ER and the central electron-dense dot was thought to be 
an artefact. Waigmann et al. (1997) have recently observed plasmodesmata in the trichomes of Nicotiana clevelandii in which the central electron-dense dot is not visible in the desmotubule. They also suggested that in that system the desmotubule may be a proteinaceous cylinder with a lumen open for transport, possibly directly connected to the cytoplasm rather than the ER lumen.

The model of Robards (1971) was put into doubt, and that of Lopez-Saez et al. was given strong support by images of tannic acid/ferric chloride-stained plasmodesmata in which the electron-lucent layer of the ER membrane appeared continuous with the electron-lucent region in the central axial structure of the plasmodesmata (Fig. 2A; Overall et al. 1982). The dimensions of the electron-dense dot in the centre of the plasmodesmata are comparable to the dimensions of appressed inner leaflets (or phospholipid head groups) of ER membrane and the central electron-lucent ring has the same dimension as the electron-lucent layer in the ER membrane (or combined hydrocarbon tails of the lipid bilayer; Overall et al. 1982). On the basis of computer-enhanced images, Ding et al. (1992) and Botha et al. (1993) proposed that this central electron-dense dot represented proteinaceous particles, but they did not reconcile this suggestion with considerations of the staining pattern expected for the phospholipid head groups of the inner leaflet of the ER membrane bilayer, nor of what the staining pattern would be when the desmotubule is expanded. They also identified fine electron-dense strands which crossed the electron-lucent layer of the desmotubule. At the necks of plasmodesmata, the electron-lucent ring of the ER increases its diameter, as does the central black dot as it opens into the lumen of the ER (Fig. 3A-D). Occasionally, the electron-lucent layer at the neck region of the desmotubule can be observed surrounded by an electron-dense layer of the dimensions expected for the outer phospholipid head groups of the ER (Fig. 3B). The absence of the mottled layer or electrondense particles embedded in the ER membrane, as proposed by Ding et al. (1992), suggests that they are not integral to the desmotubule structure. Contrary to earlier ideas about lipid packing, it has been shown that lipids similar to those in ER would be able to pack into a structure with a tight curvature like that required in the desmotubule (Overall et al. 1982).

Tilney et al. (1991) concluded that the central axial structure in the plasmodesmata of gametophytes of Onoclea was composed mainly of proteins, as digestion with a protease removed the structure but it remained intact following treatment with detergents. They proposed that the desmotubule develops as a tightly furled ER membrane and that intramembranous proteins are transported in the plane of the ER membrane into the desmotubule region and become cross-linked, forming a stable cytoskeletallike element. At the very least they concluded that a lipid-based desmotubule must be stabilized by associated proteins. In direct contrast to these observations, however, Turner et al. (1994) showed that extraction with membrane-solubilizing detergents removed the plasma membrane and the desmotubule from the plasmodesmata of wall fragments from maize root tips. While heavy proteolysis removed the entire plasmodesma, leaving only a hole in the wall, limited proteolysis extracted material from the neck regions of the plasmodesmata but there were no discernible effects in the cores of the plasmodesmata. It is difficult to reconcile these conflicting observations, but the prediction by Tilney et al. (1991) on the basis of their model that diffusion of membrane lipids through the plasmodesmata would take place in the plasma membrane and not the desmotubule was not borne out by results of Grabski et al. (1993). They 
found that lipids can diffuse between cells in the ER but not in the plasma membrane, pointing to a membranous desmotubule continuous with the ER. A further observation of Tilney et al. (1991), namely that the desmotubule breaks its connection readily from the ER during plasmolysis, has also been contradicted subsequently. The fluorescent probe $\mathrm{DiOC}_{6}$ has been used to demonstrate that the ER is continuous between adjacent cells at pit fields and remains tightly associated with plasmodesmata during at least the initial stages of plasmolysis (Oparka et al. 1994).

\subsubsection{Transport Through the Desmotubule?}

While the general interpretation of the desmotubule is a tightly furled cylinder of ER that does not function as a pathway for intercellular transport (Overall et al. 1982), it must be remembered that the images of closed desmotubules have been obtained following various tissue preparation techniques which could well stimulate the closure of such a channel or, alternatively, such a channel may be opened only momentarily during specific transport. Dilated portions of desmotubules have often been observed (Fig. 2E; Robinson-Beers and Evert 1991; Glockmann and Kollmann 1996; Waigmann et al. 1997), usually in the centre of plasmodesmata. The desmotubules without central electron-dense dots observed by Waigmann et al. (1997) in Nicotiana clevelandii trichomes may well simply be an ER tubule which is not tightly furled, but rather has a central lumen continuous with the ER. It has been thought generally that constrictions in the dimensions of the plasmodesmata at the neck regions would act as a barrier to the opening of the desmotubule, but it now appears that this neck constriction is an artefact (Radford et al. 1998). The potential for ER to act as intercellular channel for photosynthates (Gamalei et al. 1994) or water (Zhang and Tyerman 1997) has refocused interest on the possible role of ER in intercellular transport. Lazzaro and Thomson (1996) have observed a dynamic vacuolar-tubular network staining with Lucifer Yellow $\mathrm{CH}$ in trichomes of chickpea that is connected between adjacent cells via fluorescent strands across the intervening cell wall. They'proposed that the vacuolar-tubular network in that system was continuous with the desmotubule and that intercellular transport of its contents occurred via a lumen in the desmotubule. The desmotubule may be a dynamic continuity of intracellular membranous networks such as ER or vacuolar-tubular systems which make wholesale movements through the plasmodesmata, possibly powered by an actin-myosin system.

\subsection{Cytoplasmic Annulus}

The cytoplasmic annulus is defined as the space between the desmotubule and the plasma membrane and it has been thought to be the major pathway for transport of molecules from cell to cell (Gunning and Overall 1983). There is continuing controversy over the details of any molecular components within the cytoplasmic annulus and the dimensions and geometry of the cytoplasmic annulus that is available for transport. 


\subsubsection{Interpretation of the Mottled Layer Outside the Desmotubule:}

a Possible Role for Actin

The mottled layer surrounding the electron-lucent layer of the desmotubule shows various patterns of electron-dense and electron-lucent regions. The interpretation of the architecture of this region has been controversial largely because of uncertainties about the mode of action of stains. Often this mottled layer abuts directly onto the plasma membrane, as in Fig. 2B, but when the cytoplasmic annulus is expanded, there is an electron-lucent region between the mottled layer and the plasma membrane, as in Fig. 2C.

The interpretation of the mottled layer as the outer wall of the desmotubule (Robards 1971; Olesen 1979) has largely been discounted due to the arguments presented above. Overall et al. (1982) suggested that the electron-lucent regions in the mottled layer of plasmodesmata from Azolla roots may represent negatively stained particles. In some transverse sections of plasmodesmata, one or possibly two adjacent electronlucent regions appear clearly as circles $4.5 \mathrm{~nm}$ in diameter and outlined by electrondense material (Fig. 2B, C). These electron-lucent circles appear to be spheres, as three such electron-lucent circles were visible in one particularly fortuitous longitudinal oblique section of a plasmodesma (Fig. 2D). The fact that all of these spheres are not clearly visible in a transverse section suggests that they are not positioned side by side, but rather form some sort of spiral or other longitudinal arrangement. The occasional clearly demarcated sphere suggests either that any superimposed spheres within the section are in exact register or that it is not superimposed by another sphere within the plane of the section. Since only two of the total of nine particles thought to encircle the perimeter of the desmotubule (Overall et al. 1982) are clear, it appears that the remaining seven are superimposed by other particles within the section but out of register (Fig. 2B). This would suggest that within the section thickness, say $70 \mathrm{~nm}$, the particles make approximately one and three quarter complete spirals around the desmotubule, that is one spiral every $40 \mathrm{~nm}$. The three clear particles in Fig. 2D suggest that the oblique section of the plasmodesma was cut by chance at the angle of the spiral of particles. The spiral of material around the desmotubule of a plasmodesma from a Berberis floral nectary (Fig. 2E) may well be composed of electron-lucent particles as observed in Azolla plasmodesmata. It is interesting that striations of particles on the surface of the desmotubule at an angle of $20-30^{\circ}$ with reference to the transverse axis of the desmotubule have been proposed by Ding et al. (1992) and Botha et al. (1993). Ding et al. (1992) obtained remarkably similar images to that in Fig. 2B following freeze substitution of leaves of Nicotiana tabacum with and without tannic acid (their Fig. 6a, b). However, their transverse sections did not include any clearly demarcated electron-lucent spheres as in Fig. 2B, but they were almost identical to Figs. 12-14 in Overall et al. (1982). Perhaps the section thickness in these cases is such that the details of all the individual particles are obscured by other particles that are superimposed but out of register within the section.

Most of the images presented in Ding et al. (1992) were manipulated by computer image processing in general to enhance the "particulate nature of plasmodesmata". This image manipulation led Ding et al. (1992) to conclude that the mottled layer consisted of electron-dense particles of $3 \mathrm{~nm}$ diameter and embedded in the lipid on the cytoplasmic face of the desmotubule, with similar electron-dense particles embedded 
in the inner face of the plasma membrane. The electron-lucent regions of the mottled layer were interpreted as spaces between these electron-dense particles. However, this interpretation is difficult to reconcile with images such as Fig. 2C, in which the electron-lucent areas of the mottled layer are clearly visible outlined by a thin electrondense layer, and yet any electron-dense particles on the inner leaflet of the plasma membrane are separated from the mottled layer by an obvious cytoplasmic annulus. The mottled layers in the plasmodesmata shown in Fig. 2B, C are approximately $9 \mathrm{~nm}$ in diameter, comparable to the 10-nm diameter mottled layer observed by Ding et al. (1992). If the interpretation of Ding et al. were correct, then there would be only $3 \mathrm{~nm}$ electron-dense particles surrounding the desmotubule before the cytoplasmic annulus in plasmodesmata with an expanded cytoplasmic annulus such as in Fig. 2C. Even in the image provided by Ding et al. (1992) of a plasmodesma with an expanded cytoplasmic annulus (their Fig. 4b), however, the desmotubule is surrounded by an electron-dense or mottled layer of $10 \mathrm{~nm}$ !

Botha et al. (1993) have also used computer image enhancement on images of plasmodesmata at the Kranz mesophyll-bundle sheath interface in Themeda triandra. They presented images of these plasmodesmata where the cytoplasmic annulus was constricted as in Fig. 2B. While the false colour images they produced were intriguing, they did not provide any new insights on the structure of the mottled layer. Indeed the usefulness of computer-generated image enhancement to gain information that is not already visible in sections has to be questioned. Clearly, progress will require techniques for generating 3-D computer models of the structure of plasmodesmata based upon the known staining properties of various structures throughout the thickness of a section.

The electron-lucent particles in the mottled layer may well be actin. Using immunogold cytochemistry and rhodamine phalloidin labelling in the confocal microscope, White et al. (1994) demonstrated that actin is a component of plasmodesmata not only at their necks but also along their length. Actin filaments that have been chemically fixed in the presence of $0.2 \%$ tannic acid appear as electron-lucent particles of 5.5-7 nm diameter surrounded by an electron-dense coat making their total diameter 11-17 nm (Maciver et al. 1991). These dimensions are comparable to the dimensions quoted for the electron-lucent particles and surrounding electron-dense material in the mottled layer (Overall et al. 1992). Usually only one or possibly two gold particles were observed in any transverse section of a plasmodesma. As the antibody is able to recognize only actin that is at the surface of the section, this observation is consistent with the suggestion that the electron-lucent particles form a spiral around the desmotubule. The close association of actin, ER, and the plasma membrane has been observed previously (Lichtscheidl et al. 1990) and in retrospect it is not surprising that it is also present in the plasmodesmata.

\subsubsection{Spokes Connect the Mottled Layer with the Plasma Membrane}

Electron-dense spokes have been observed to connect the mottled layer to the inner leaflet of the plasma membrane where there is a clear electron-lucent cytoplasmic annulus (Fig. 2C). These spokes have been observed in a wide variety of plasmodesmata in transverse (e.g. Burgess 1971; Tilney et al 1991; Ding et al. 1992; Schulz 1995; Cook 
et al. 1997) and longitudinal sections (Ding et al. 1992). The spokes are clearly visible in expanded cytoplasmic annuli, although it is possible that they are also present in a shortened form or parallel to the axis of the plasmodesma when no clear electron lucent cytoplasmic annulus can be seen. These spokes may be myosin which is expected to have a close association with ER (Liebe and Quader 1994) and actin microfilaments, and in addition there is high ATPase activity at the plasmodesmata (Robards and Lucas 1990). Indeed, myosin has recently been identified in the plasmodesmata of the green alga Chara corallina (Blackman and Overall 1998) and higher plants (Radford and White 1998).

\subsubsection{Dimensions and Regulation of the Transport Pathway Through the Cytoplasmic Annulus}

Since the cytoplasmic annulus was thought to be the major pathway for intercellular transport of molecules (Gunning and Overall 1983), much attention has been focused on the proposed dimensions of the pathways available for transport. Overall et al. (1982) argued that even though plasmodesmata may have expanded cytoplasmic annuli in the centre, in general there was a tight association of the plasma membrane to the mottled layer at the neck region in a construction termed the neck constriction. Transport was proposed to be restricted to channels surrounding electron-lucent particles arranged side by side in the mottled layer. These spaces were calculated to be in the order of $3 \mathrm{~nm}^{2}$, comparable to the then known size exclusion limit for plasmodesmata in the order of $1-\mathrm{kDa}$ (Goodwin 1983; Tucker 1982). If it is accepted that these particles are arranged in a spiral, then the transport pathway would form a spiral of at least the thickness of the particles, approximately $6 \mathrm{~nm}$.

In a very thorough study, Schulz (1995) demonstrated that a 1-h treatment of Pisum sativum roots with $350 \mathrm{mM}$ mannitol, a treatment known to transiently stimulate phloem unloading in this system, dramatically increased the width of plasmodesmata compared with those in roots not treated with mannitol, or those treated with mannitol for $3 \mathrm{~h}$. In all treatments, the dimensions of the desmotubule and the associated mottled layer remained constant and it was the cytoplasmic annulus that increased dramatically with the short mannitol treatment, suggesting that this pathway was used for transport during phloem unloading. The spokes connecting the mottled layer to the plasma membrane remained intact during the widening of the cytoplasmic annulus. These findings corroborated the conclusion of Bret-Harte and Silk (1994) that sucrose flow through plasmodesma pore sizes of $3 \mathrm{~nm}^{2}$ was not sufficient to meet the carbon demands of root tips.

Subsequently, it has been demonstrated that the neck constrictions in the roots of Allium cepa L. are an artefact of callose deposition around the neck stimulated by tissue preparation and chemical fixation (Radford et al. 1998). Callose is a $\beta$-1, 3-glucan which is rapidly deposited between the plasma membrane and the wall in response to wounding (Sec. 5.4.2). In roots treated with 2-deoxy-D-glucose (DDG), an inhibitor of callose synthesis, prior to and during fixation, the plasmodesmata have open funnelshaped necks, but the plasmodesmata in roots prepared without DDG had clear neck constrictions (Radford et al. 1998). The open neck configuration seen in Fig. 2C is in a plasmodesma of an Egeria densa leaf treated with DDG. It maybe that the 1-h manni- 
tol treatment in the work of Schulz (1995) served to protect the plasmodesmata from the damaging effects of chemical fixation.

In any event, the width of the cytoplasmic lumen in these open plasmodesmata between the mottled layer and the plasma membrane calculated from the images and measurements in Schulz (1995) and Radford et al. (1998) is 7-14 nm. If the mottled layer represents a spiral of particles, then the pathway available for transport through the cytoplasmic lumen between the particles could be $13-20 \mathrm{~nm}$ wide! This pathway would clearly allow passage of molecules much larger than $1 \mathrm{kDa}$. In recent years, some cells have been shown to have much larger size exclusion limits. For example, dextrans of at least $10 \mathrm{kDa}$ pass between companion cells and sieve elements (Kempers and van Bel 1997), 7-kDa dextrans pass plasmodesmata in tobacco trichomes (Waigmann and Zambryski 1995), and 40-kDa molecules pass plasmodesmata in $\mathrm{Ni}$ tella (Kikuyama et al. 1992). Furthermore, virally encoded proteins assist the intercellular passage of macromolecules up to $30-\mathrm{kDa}$ (for review, see Ghoshroy et al. 1997). Certainly molecules with a Stokes radius of larger than the $3 \mathrm{~nm}$ calculated for a 20 $\mathrm{kDa}$ dextran (Wolf and Lucas 1994) would be expected to pass the cytoplasmic channel.

If this larger cytoplasmic annulus is to be considered the in vivo state of transporting plasmodesmata, the question arises as to why molecules larger than $1 \mathrm{kDa}$ do not routinely pass through plasmodesmata. One possibility is that the techniques used to study plasmodesmal transport such as microinjection stimulate a wound response and close the plasmodesmata. A second possibility is that there is some as yet unidentified molecular sieve at the necks of plasmodesmata that regulates the intercellular passage of molecules. Such a proposal is supported by the observation that plasmodesmata actually pass larger molecules following severe plasmolysis (Erwee and Goodwin 1984), suggesting that some delicate molecular sieving mechanism at the neck may have been damaged.

A third possibility is that the dimensions of this pathway are constantly being modified. A possible site for this modulation could be around the neck region where electron-dense material has been observed connecting the plasma membrane and the ER as it enters the plasmodesma (Fig. 3G). Intercellular communication is inhibited with increasing intracellular concentration of calcium (Erwee and Goodwin 1983) and inositol 1, 4, 5-triphosphate (Tucker 1988), and in some systems increased with increases in intracellular ATP (Tucker 1993; Cleland et al. 1994). This suggests that a contractile calcium-binding protein may be involved in the regulation of intercellular communication. Indeed, the calcium-binding phosphoprotein centrin which forms filamentous structures that contract rapidly with increases in calcium concentration and require ATP for extension (Salisbury 1995) appears to be localized to the neck regions of plasmodesmata (Blackman et al. 1999). Centrin may modulate the pathway for transport through the cytoplasmic sleeve by controlling the space between the plasma membrane and the ER at the neck region.

Alternatively, the dimensions of the pathways through the plasmodesmata may be regulated by an actin-myosin system. Treatment with the actin-disrupting agent, cytochalasin B, dramatically increased the dimensions of the cytoplasmic annulus in one of the three species studied (White et al. 1994), suggesting that actin may play a role in maintaining the precise dimensions of this transport pathway through plasmodesmata. Perturbation of the actin filaments in tobacco mesophyll cells by injections of cyto- 
chalasin D or profilin allowed the intercellular passage of a $20-\mathrm{kDa}$ dextran normally excluded (Ding et al. 1996). The increase in dimensions of plasmodesmata observed following papain treatment (Tilney et al. 1991) may have been due to disruption of the actin and myosin involved in maintaining integrity of the pathway.

\subsection{Specializations of the Wall Sleeve}

The sleeve of wall material surrounding the plasmodesmata must be quite specialized in order to accommodate, or even modulate, changes in dimensions of plasmodesmata such as described above. There is often an electron-lucent region in the wall surrounding the plasmodesmata (Figs. $2 \mathrm{E}$ and $3 \mathrm{H}$ ). Such electron-lucent wall sleeves are not visible in other longitudinal sections of plasmodesmata (Figs. 2A, D, 3E-G) because in these preparations the walls had been digested out after fixation in order to enhance the details of the plasmodesma substructure.

\subsubsection{Plasmodesmata Are Firmly Anchored to the Surrounding Wall}

Fine electron-dense spokes are sometimes observed to connect the plasma membrane to the surrounding wall material (Fig. 3H; Cook et al. 1997) clearly at the neck region but also down the length of the plasmodesma. These connections presumably anchor the plasmodesmata in the walls following plasmolysis (Tilney et al. 1991; Oparka et al. 1994) or wall isolation (Tilney et al. 1991; Turner et al. 1994). Of course, they may also be involved in regulating the dimensions of the plasmodesmal channel.

\subsubsection{Callose}

Callose, a $\beta$-1,3-glucan, is deposited between the plasma membrane and the plant wall and appears electron-lucent in electron micrographs (for review, see Stone and Clarke 1992). Callose is deposited at plasmodesmata in response to wounding or chemical fixation (Smith and McCully 1977; Galway and McCully 1987; Northcote et al. 1989; Balestrini et al. 1994; Turner et al. 1994; Radford et al. 1998), and Delmer et al. (1993) have localized the callose synthase to plasmodesmata. The deposition of callose around plasmodesmata has been thought to restrict transport through them (Olesen and Robards 1990), thereby serving as a crude sphincter mechanism which is deposited very rapidly but takes hours to disappear. One possible site of callose deposition is the raised electron-lucent collar surrounding the necks of some plasmodesmata (Olesen and Robards 1990). This collar is associated with constricted necks within the plasmodesmata (see Radford et al. 1998). Turner et al. (1994) found that antibodies to callose did not label these raised collars but rather the wall regions between them. In addition, following treatment with callose-degrading enzymes there was no labelling of callose, but the raised collars were not removed. Turner et al. (1994) concluded that the cell wall around the plasmodesmata was structurally subdivided, with an inner amorphous collar surrounded by a peripheral zone in which callose is interspersed with the fibrillar wall material. On the basis of differential extractions, they concluded that the 
inner amorphous zone was a carbohydrate held in position by protein links. This contradicts the findings of Radford et al. (1998) in which inhibition of callose deposition with DDG prevented the formation of raised collars.

\subsubsection{Pectin}

Pectins play a role in cell wall hydration, adhesion of adjoining cells, and wall plasticity (Vreeland et al. 1989). Unesterified pectins which are found in the middle lamella are located to the plasmodesmata in tomato pericarp cells (Casero and Knox 1995). Low esterified pectin surrounds the plasmodesmata in ripening apples and are not involved in calcium cross-bridging, but are probably surrounded by a cationic environment (Roy et al. 1997). These modifications are thought to protect the wall around the plasmodesmata from hydrolytic enzyme breakdown during ripening. Similar cellulase-resistant wall tubes have been observed around barley aleurone plasmodesmata (Taiz and Jones 1973). During protoplast formation, cells may remain attached at plasmodesmata (for review, see Carr 1976), suggesting that this protective wall sleeve may be a common feature of plasmodesmata. It remains to be demonstrated if and how this pectin sleeve plays a role in modifying the dimensions of the plasmodesmal pathway.

\subsubsection{Putative Extracellular Sphincters}

Inclusion of tannic acid in the fixative has highlighted a number of presumably proteinaceous components just outside the plasma membrane of the plasmodesmata (Figs. $2 \mathrm{D}, 3 \mathrm{E}-\mathrm{G})$. They have now been observed by a number of workers in a variety of plant tissues (Olesen 1979; Overall et al. 1982; Mollenhauer and Morré 1987; Olesen and Robards 1990; Tilney et al. 1991; Robinson-Beers and Evert 1991; Badelt et al. 1994; Cook et al. 1997) and can also be discerned, although not as clearly, in material prepared without chemical fixation or tannic acid (Badelt et al. 1994). Rings of electrondense material encircle the necks of some plasmodesmata and occasionally they can be clearly discerned to comprise definite particles (Fig. 3E) which, in turn, appear to have a clear substructure (Fig. 3F). Sometimes the ring is closely associated with the plasma membrane (Fig. 2C). In other images, the ring is expanded out from the plasma membrane and connected to it via spokes or strings (Figs. 3E, F). This arrangement of particles is reminiscent of the open and closed rings of particles observed by Willison (1976) around the necks of plasmodesmata following freeze fracture. A second major structure observed is formed by electron-dense spirals which encircle the length of the plasmodesma (Fig. 3G), possibly anchoring at the ring of particles or on the plasma membrane around the necks of the plasmodesma (Badelt et al. 1994).

Nothing is known of the molecular composition of these structures. Olesen and Robards (1990) suggested that the electron-dense particles may be callose synthase complexes, but this is contradicted by the deposition of callose in the root cap cells of onion roots in which these electron-dense particles are absent (Radford et al. 1998). The particle rings and spiral, either individually or together, could contract to constrict the cytoplasmic annulus. Alternatively, if the spiral were a fixed length, it could be made to wrap more tightly or loosely around the plasmodesma if its ends at the 


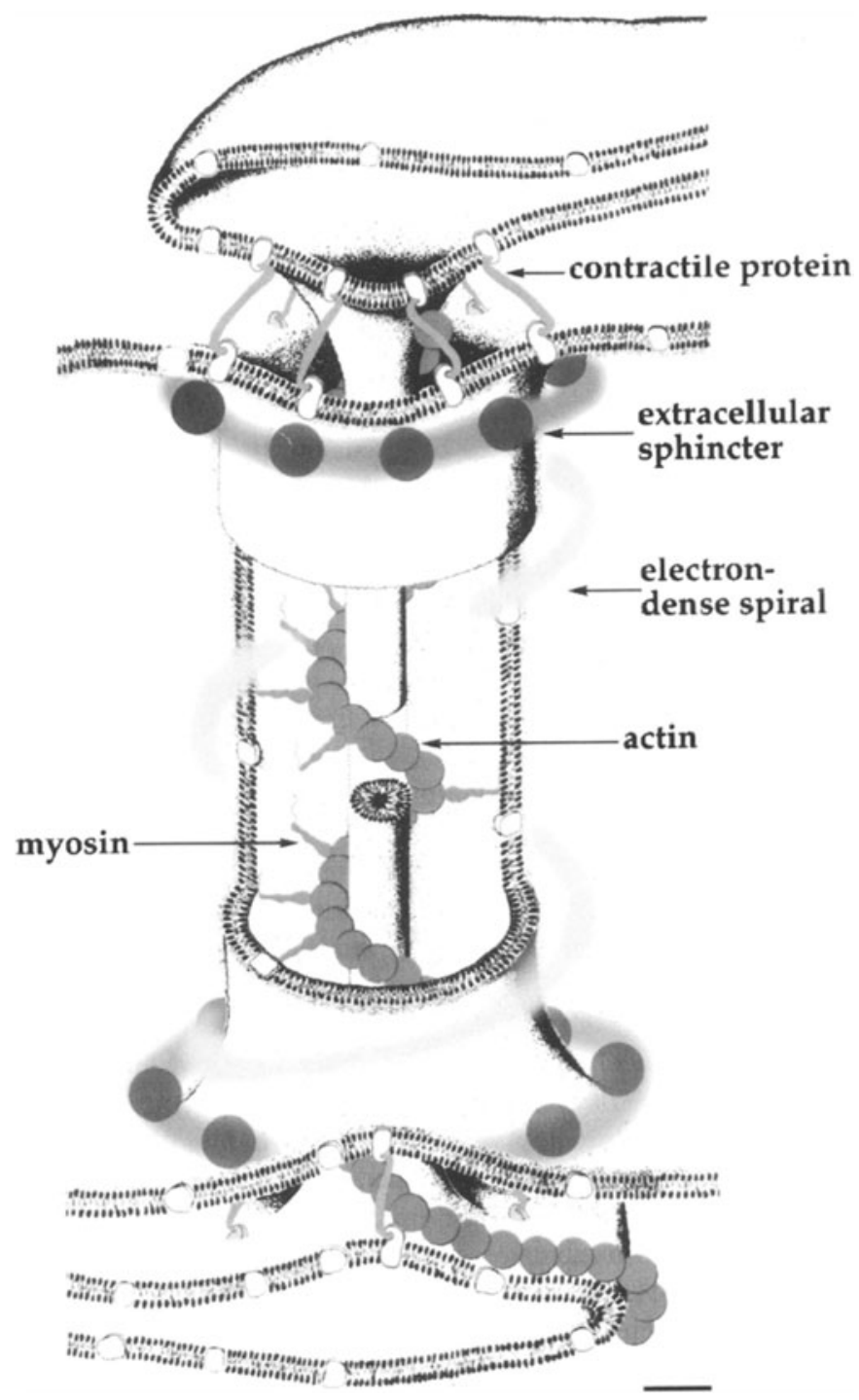

Fig 4. Model of a plasmodesma. Actin and myosin, which are helically arranged around the desmotubule, connect the plasma membrane to the desmotubule. Contractile proteins (possibly centrin) link the plasma membrane to the endoplasmic reticulum (ER) via anchoring proteins at the neck of the plasmodesma. These anchoring proteins within the membrane may have a close association with the putative extracellular sphincter. These extracellular structures may provide anchoring points to the wall for the components of the plasmodesma. The extracellular sphincters at the neck and the extracellular spiral could also be involved in modulating the outer dimensions of the plasmodesma. Transport of specific macromolecules could occur via the actin-myosin motile system or via a dilated ER. The size exclusion limit for possible transport between cells would depend on the dimension of the gap between the desmotubule and the plasma membrane as determined by contractile proteins, or by an as yet unidentified molecular sieve. Bar $10 \mathrm{~nm}$. (Overall and Blackman 1996) 
necks of the plasmodesma were wound in opposite directions. This could occur by a fixed attachment to the ring of particles which could spin in opposite directions driven by a molecular motor. Alternatively, the ring of particles could remain fixed and the point of attachment of the spiral could move around the rings in opposite directions at each end of the plasmodesma. There is a difficulty in considering the molecular motors operating in the apoplasm, and it may be that the ring of particles themselves are transmembrane proteins as in Fig. 3G or are connected to transmembrane proteins. The cytochalasin treatment that expanded plasmodesmata also caused the loss of these extracellular structures (White et al. 1994), suggesting that the actin-myosin complex is connected in some way via transmembrane components to the wall sleeve. A more radical proposal would be that these extracellular components actually contain actin. The high ATPase concentration in the plasmodesmata (Robards and Lucas 1990) may be involved in powering the dynamics of any proposed motor, but until the molecular details of these structures are resolved, these ideas must remain as fanciful speculation!

\section{$6 \quad$ Concluding Remarks}

The static models of plasmodesmata generated largely on the basis of electron microscopy (e.g. Gunning and Overall 1983) must now incorporate the emerging molecular information about plasmodesmal components. Fig. 4 is an attempt to do this, incorporating the various components of plasmodesmal substructure presented in this chapter. This model is no doubt a gross oversimplification of what could well turn out to be a complex interaction of as yet unknown molecular components. Further progress in our understanding of the substructure of plasmodesmata and how these assemblages function in the transport of viruses and macromolecules awaits further molecular characterization of these components.

\section{References}

Badelt K, White RG, Overall RL, Vesk M (1994) Ultrastructural specialisations of the cell wall sleeve around plasmodesmata. Am J Bot $81: 1422-1427$

Balestrini R, Romera C, Puigdomenech P, Bonfante P (1994) Location of a cell-wall hydroxyprolinerich glycoprotein, cellulose and beta-1, 3-glucans in apical and differentiated regions of maize mycorrhizal roots. Planta 195:201-209

Blackman LM, Harper JDI, Overall RL (1999) Localization of a centrin-like protein to higher plant plasmodesmata. Eur J Cell Biol 78:297-304

Blackman LM, Overall RL (1998) Immunolocalisation of the cytoskeleton to plasmodesmata of Chara corallina. Plant J 14:733-741

Botha CEJ, Hartley BJ, Cross RHM (1993) The ultrastructure and computer-enhanced digital image analysis of plasmodesmata at the Kranz mesophyll-bundle sheath interface of Themeda triandra var. imberbis (Retz) A. Camus in conventionally fixed leaf blades. Ann Bot 72:255-261

Bret-Harte MS, Silk WK (1994) Nonvascular, symplastic diffusion of sucrose cannot satisfy the carbon demands of growth in the primary root tip of Zea mays L. Plant Physiol 105:19-33

Burgess J (1971) Observations on structure and differentiation in plasmodesmata. Protoplasma 73 : 83-95

Buvat R (1957) L'infrastructure des plasmodesmes et la continuité des cytoplasmes. C R Acad Sci Paris D 245:198-201

Carr DJ (1976) Plasmodesmata. In: Gunning BES, Robards AW (eds) Intercellular communication in plants: studies on plasmodesmata. Springer, Berlin Heidelberg New York, pp 243-289 
Casero PJ, Knox JP (1995) The monoclonal antibody JIM5 indicates patterns of pectin deposition in relation to pit fields at the plasma-membrane-face of tomato pericarp cell walls. Protoplasma 188: 133-137

Cleland RE, Fujiwara T, Lucas WJ (1994) Plasmodesmal-mediated cell-to-cell transport in wheat roots is modulated by anaerobic stress. Protoplasma 178:81-85

Cook ME, Graham LE, Botha CEJ, Lavin CA (1997) Comparative ultrastructure of plasmodesmata of Chara and selected bryophytes: toward an elucidation of the evolutionary origin of plant plasmodesmata. Am J Bot 84:1169-1178

Delmer DP, Volokita M, Solomon M, Fritz U, Delphendahl W, Herth W (1993) A monoclonal antibody recognises a $65-\mathrm{kDa}$ higher plant membrane polypeptide which undergoes cation-dependent association with callose synthase in vitro and colocalizes with sites of high callose deposition in vivo. Protoplasma 176:33-42

Ding B, Turgeon R, Parthasarathy MV (1992) Substructure of freeze-substituted plasmodesmata. Protoplasma 169:28-41

Ding B, Kwon M-O, Warnberg L (1996) Evidence that actin filaments are involved in controlling the permeability of plasmodesmata in tobacco mesophyll. Plant J 10:157-164

Erwee MG, Goodwin PB (1983) Characterisation of Egeria densa Planch. leaf symplast: inhibition of the intercellular movement of fluorescent probes by group II ions. Planta 158:320-328

Erwee MG, Goodwin PB (1984) Characterisation of Egeria densa symplast: response to plasmolysis, deplasmolysis and to aromatic amino acids. Protoplasma 122:162-168

Fleurat-Lessard P, Bouché-Pillon S, Leloup C, Lucas WJ, Serrano R, Bonnemain JL (1995) Absence of plasma membrane $\mathrm{H}^{+}$-ATPase in plasmodesmata located in pit-fields of the young reactive pulvinus of Mimosa pudica L. Protoplasma 188:180-185

Galway ME, McCully ME (1987) The time course of the induction of callose in wounded pea roots. Protoplasma 139:77-91

Gamalei YV, van Bel AJE, Pakhova MV, Sjutkina AV (1994) Effects of temperature on the conformation of the endoplasmic reticulum and on starch accumulation in leaves with the symplasmic minor-vein configuration. Planta 194:443-453

Ghoshroy S, Lartey R, Sheng J, Citovsky V (1997) Transport of proteins and nucleic acids through plasmodesmata. Annu Rev Plant Physiol Plant Mol Biol 48:27-50

Glockmann C, Kollmann R (1996) Structure and development of cell connections in the phloem of Metasequoia glyptostroboides needles I. Ultrastructural aspects of modified primary plasmodesmata in Strasburger cells. Protoplasma 193:191-203

Goodwin PB (1983) Molecular size limit for movement in the symplast of the Elodea leaf. Planta 157: 124-130

Grabski S, de Feijter AW, Schindler M (1993) Endoplasmic reticulum forms a dynamic continuum for lipid diffusion between contiguous soybean root cells. Plant Cell 5:25-38

Gu X, Verma DPS (1996) Phragmoplastin, a dynamin-like protein associated with cell plate formation in plants. EMBO J 15:695-704

Gunning BES (1978) Age-related and origin-related control of the numbers of plasmodesmata in cell walls of developing Azolla roots. Planta 143:181-190

Gunning BES, Overall RL (1983) Plasmodesmata and cell-to-cell transport in plants. Bioscience 33: 260-265

Hepler PK (1982) Endoplasmic reticulum in the formation of the cell plate and plasmodesmata. Protoplasma 111:121-133

Jones MJK (1976) The origin and development of plasmodesmata. In: Gunning BES, Robards AW (eds) Intercellular communication in plants: studies on plasmodesmata. Springer Verlag, Berlin Heidelberg New York, pp 81-103

Kempers R, van Bel AJE (1997) Symplasmic connections between sieve element and companion cell in the stem phloem of Vicia faba L. have a molecular exclusion limit of at least 10-kDa. Planta 201: 195-210

Kikuyama M, Hara Y, Shimada K, Yamamoto K, Hiramoto Y (1992) Intercellular transport of macromolecules in Nitella. Plant Cell Physiol 33:413-417

Lazzaro MD, Thomson WW (1996) The vacuolar-tubular continuum in living trichomes of chickpea (Cicer arietinum) provides a rapid means of solute delivery from base to tip. Protoplasma 193: 181-190

Lichtscheidl IK, Lancelle SA, Hepler PK (1990) Actin-endoplasmic reticulum complexes in Drosera. Their structural relationship with the plasmalemma, nucleus, and organelles in cells prepared by high pressure freezing. Protoplasma 155:116-126

Liebe S, Quader H (1994) Myosin in onion (Allium cepa) bulb scale epidermal cells: involvement in dynamics of organelles and endoplasmic reticulum. Physiol Plant 90:114-124

Lopez-Saez JF, Gimenez-Martin G, Risueno MC (1966) Fine structure of the plasmodesm. Protoplasma $61: 81-84$ 
Maciver SK, Wachsstock SH, Schwarz WH, Pollard TD (1991) The actin filament severing protein actophorin promotes the formation of rigid bundles of actin filaments crosslinked with alpha-actinin. J Cell Biol 115: 1621-1628

Marchant HJ (1976) Plasmodesmata in algae and fungi. In: Gunning BES, Robards AW (eds) Intercellular communication in plants: studies on plasmodesmata. Springer, Berlin Heidelberg New York, pp 59-78

Mollenhauer H, Morré J (1987) Some unusual staining properties of tannic acid in plants. Histochemistry $88: 17-22$

Northcote DH, Davey R, Lay J (1989) Use of antisera to localise callose, xylan and arabinogalactan in the cell-plate, primary and secondary walls of plant cells. Planta 178:353-366

O'Brien TP, Thimann KV (1967) Observations on the fine structure of the oat coleoptile. II. The parenchyma cells of the apex. Protoplasma 63:417-442

Olesen P (1979) The neck constriction in plasmodesmata. Evidence for a peripheral sphincter-like structure revealed by fixation with tannic acid. Planta $144: 349-358$

Olesen P, Robards AW (1990) The neck region of plasmodesmata: general architecture and some functional aspects. In: Robards AW, Lucas WJ, Pitts JD, Jongsma HJ, Spray DC (eds) Parallels in cell to cell junctions in plants and animals. Springer, Berlin Heidelberg New York, pp 145-170

Oparka KJ, Prior AM, Crawford JW (1994) Behaviour of plasma membrane, cortical ER and plasmodesmata during plasmolysis of onion epidermal cells. Plant Cell Environ 17:163-171

Oparka KJ, Boevink P, Santa Cruz S (1996) Studying the movement of plant viruses using green fluorescent protein. Trends Plant Sci 1:403-441

Oparka KJ, Prior DAM, Santa Cruz S, Padgett HS, Beachy RN (1997) Gating of epidermal plasmodesmata is restricted to the leading edge of expanding infection sites of tobacco mosaic virus (TMV). Plant J 12:781-789

Overall RL, Blackman LM (1996) A model of the macromolecular structure of plasmodesmata. Trends Plant Sci 1:307-311

Overall RL, Wolf J, Gunning BES (1982) Intercellular communication in Azolla roots I. Ultrastructure of plasmodesmata. Protoplasma 111:134-150

Radford JE, White RG (1998) Localization of a myosin-like protein to plasmodesmata. Plant J 14: 743-750

Radford JE, Vesk M, Overall RL (1998) Callose deposition at plasmodesmata. Protoplasma 201 :30-37

Robards AW (1971) The ultrastructure of plasmodesmata. Protoplasma 72:315-323

Robards AW (1976) Plasmodesmata in higher plants. In: Gunning BES Robards AW (eds) Intercellular communication in plants: studies on plasmodesmata. Springer, Berlin Heidelberg New York, pp 15-53

Robards AW, Lucas WJ (1990) Plasmodesmata. Annu Rev Plant Physiol Mol Biol 41:369-419

Robinson-Beers K, Evert RF (1991) Fine structure of plasmodesma in mature leaves of sugarcane. Planta 184:308-318

Roy S, Watada AE, Wergin WP (1997) Characterisation of the cell wall microdomain surrounding plasmodesmata in apple fruit. Plant Physiol 144:539-547

Salisbury JL (1995) Centrin, centrosomes and mitotic spindle poles. Curr Opin Cell Bio 7:39-45

Schulz A (1995) Plasmodesmal widening accompanies the short-term increase in symplasmic phloem unloading in pea root tips under osmotic stress. Protoplasma 188:22-37

Smith MM, McCully ME (1977) Mild temperature "stress" and callose synthesis. Planta 136:65-70

Staehelin LA, Hepler PK (1996) Cytokinesis in higher plants. Cell 84:821-824

Stone BA, Clarke AE (1992) Chemistry and biology of (1-3)-b-glucans. La Trobe University Press, Melbourne, Australia

Taiz L, Jones RL (1973) Plasmodesmata and an associated cell wall component in barley aleurone tissue. Am J Bot 60:67-75

Tangl E (1879) öber offene Kommunikation zwischen den Zellen des Endosperms einiger Samen. Jb Wiss Bot 12:170-190

Thomson WW, Platt-Aloia K (1985) The ultrastructure of plasmodesmata of the salt glands of Tamarix as revealed by transmission and freeze-fracture electron microscopy. Protoplasma 125:13-23

Tilney LG, Cooke TJ, Connelly PS, Tilney MS (1991) The structure of plasmodesmata as revealed by plasmolysis, detergent extraction, and protease digestion. J Cell Sci 112:739-747

Tucker EB (1988) Inositol bisphosphate and inositol trisphosphate inhibit cell-to-cell passage of carboxyfluorescein in staminal hairs of Setcresea purpurea. Planta 174:358-363

Tucker EB (1982) Translocation in the staminal hairs of Setcreasea purpurea I. A study of the cell ultrastructure and cell-to-cell passage of molecular probes. Protoplasma 113:193-210

Tucker EB (1993) Azide treatment enhances cell-to-cell diffusion in staminal hairs of Setcreasea purpurea. Protoplasma 174:45-49

Turner A, Wells B, Roberts K (1994) Plasmodesmata of maize root tips: structure and composition. J Cell Sci 107:3351-3361 
Vaughn KC, Hoffman JC, Hahn MG, Staehelin LA (1996) The herbicide dichlobenil disrupts cell plate formation: immunogold characterisation. Protoplasma 194:117-132

Vreeland V, Morse DR, Robichaux RH, Millar KL, Hau S-ST, Laetsch WM (1989) Pectate distribution and esterification in Dubautia leaves and soybean nodules, studies with fluorescent hybridisation probe. Planta 177:435-446

Waigmann E, Zambryski P (1995) Tobacco mosaic virus movement protein-mediated protein transport between trichome cells. Plant Cell 7:2069-2079

Waigmann E, Turner A, Peart J, Roberts K, Zambryski P (1997) Ultrastructural analysis of leaf trichome plasmodesmata reveals major differences from mesophyll plasmodesmata. Planta 203: 75-84

White RG, Badelt K, Overall RL, Vesk M (1994) Actin associated with plasmodesmata. Protoplasma $180: 169-184$

Willison JHM (1976) Plasmodesmata: a freeze-fracture view. Can J Bot 54:2842-2847

Wolf S, Lucas WJ (1994) Virus movement proteins and other molecular probes of plasmodesmal function. Plant Cell Environ 17:573-585

Zambryski P (1995) Plasmodesmata: plant channels for molecules on the move. Science 270: 1943-1944

Zhang WH, Tyerman SD (1997) Effect of low oxygen concentration on the electrical properties of cortical cells of wheat roots. J Plant Physiol 150:567-572 